\title{
Microsymposium
}

MS107.002

\section{XRPD to Characterize Disordered and Amorphous Pharmaceutical Samples}

A. Newman $^{1}$

${ }^{1}$ Crystal Pharmatech and Seventh Street Development Group, Lafayette, IN, USA

X-ray powder diffraction is commonly used to characterize pharmaceutical samples to identify crystalline forms as well as differentiate crystalline vs amorphous materials. Using XRPD for amorphous materials is usually limited to determining if the materials are fully amorphous or a mixture of crystalline and amorphous materials. Changes in the amorphous halos under various conditions (grinding, drying, water sorption, etc) are usually not investigated, but can give information on the system. This presentation will discuss other uses of XRPD in characterizing amorphous and disordered systems, including amorphous solid dispersions. Patterns involving nanocrystalline, amorphous, and defected samples will be presented and examples on using various methods to obtain further information from the data (pair distribution functions, pure curve resolution methods, diffuse scattering, and Rietveld analysis) will be included.

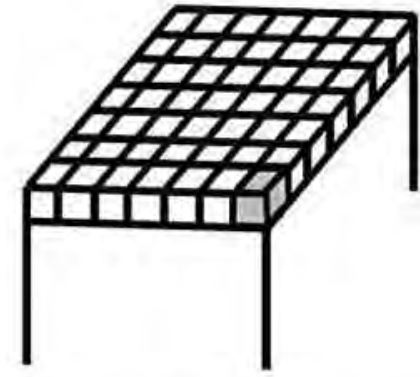

crystalline
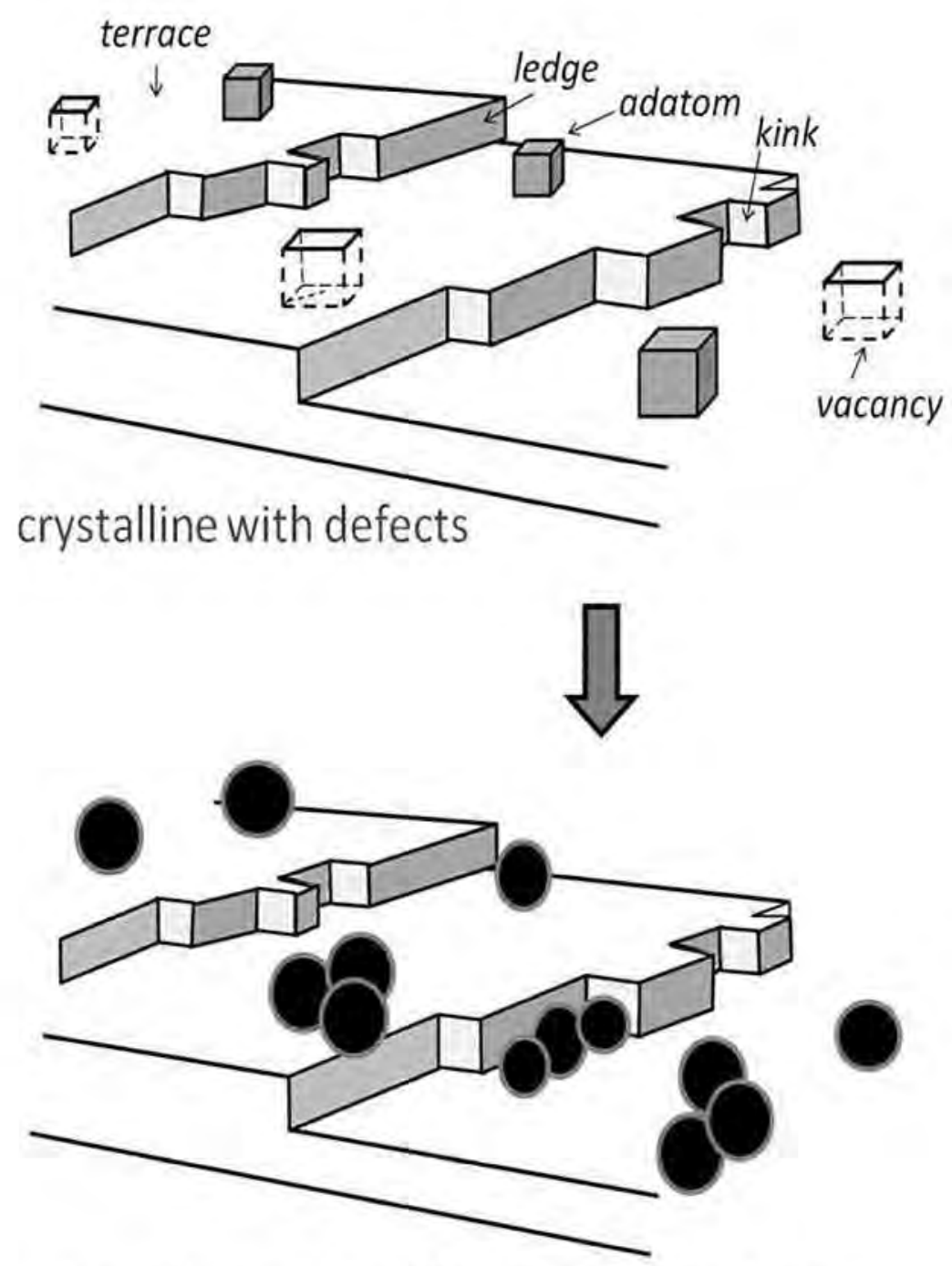

crystalline with defects and amorphous

Keywords: amorphous, disorder, pharmaceutical characterization 